\title{
ON USING THE THIN TARGET AT EXTRACTION OF PROTONS FROM THE ACCELERATORS BY BENT CRYSTAL
}

\author{
A.A.Asseev \\ Institute for High Energy Physics \\ 142284 Protvino, Moscow region, Russia.
}

\section{Abstract}

The influence of the thin target on the bent crystal extraction efficiency at $70 \mathrm{GeV}$ is discovered in the IHEP accelerator. This effect was confirmed also at $50 \mathrm{GeV}$. It is supposed that the effect can be used under a beam extraction from the higher energy accelerators.

\section{INTRODUCTION}

The effect of increasing the bent crystal extraction efficiency $[1,2]$ may be used for extraction of a beam from the high energy accelerators (e.g. Tevatron and SPS) for the new planned experiments $[3,4]$.

The estimates of results expected, due to use of thin targets, in other accelerators under extraction of protons by a bent crystal, and based on the experimetal data obtained in the $70-\mathrm{GeV}$ IHEP accelerator are given in this article.

\section{EXPERIMENTAL RESULTS}

As it was reported in [1], there is a doubling of the extracted beam intensity under extraction by the bent crystal of the $70-\mathrm{GeV}$ protons having been preliminary scattered by a thin target.

Preliminary scattering of a beam by the thin target with the following hitting the crystal by scattered particles also gives at the energy $50 \mathrm{GeV}$ an increase of extraction efficiency. However, on comparing the case of intensity doubling at extraction of the $70-\mathrm{GeV}$ protons into the beam line 14, the increase of intensity in the beam line 2 only by $30 \%$ was fixed [2]. Both of the experiments were carried out under the same geometric conditions.

Fig.1 shows the number of particles extracted from the accelerator by bent crystal versus the thin target coordinate for two levels of the primary bean energy: $70 \mathrm{GeV}$ (curve 1) and $50 \mathrm{GeV}$ (curve 2).

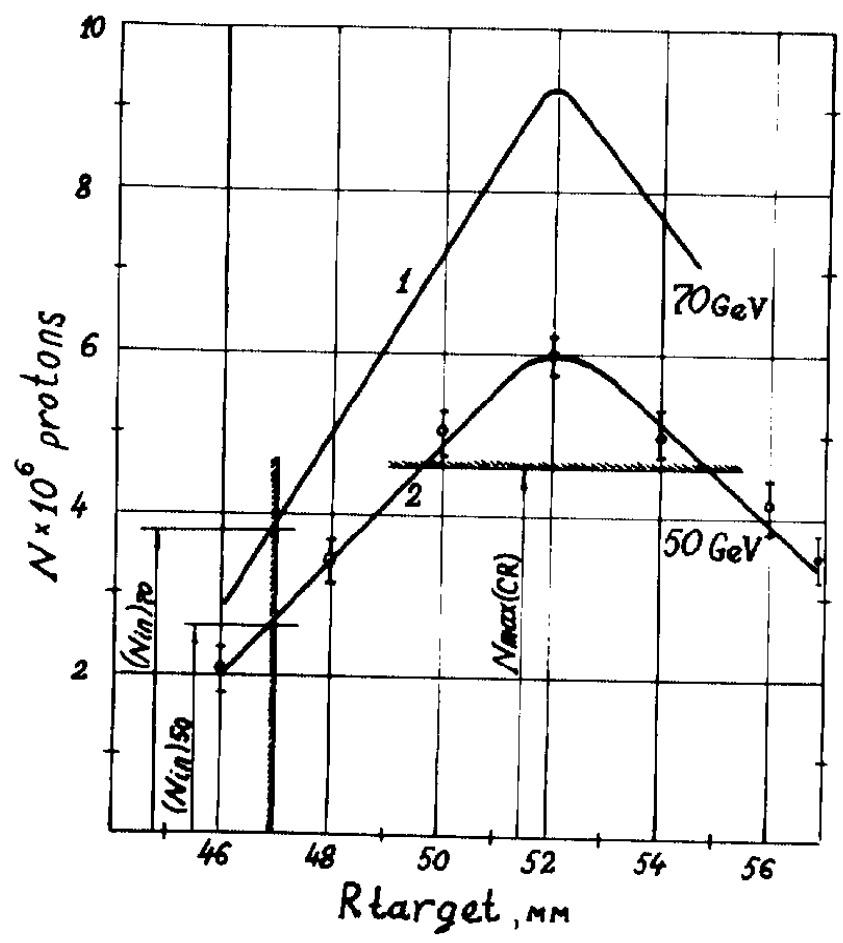

Figure 1: Number of particles extracted from the accelerator by bent crystal under preliminary beam scattering by a thin internal target versus its coordinate. Curve 1 the energy $70 \mathrm{GeV}, 2-50 \mathrm{GeV} . N_{\max }(C R)$ is a maximum number of particles extracted by bent crystal without a thin target.

Analysis of the data $[1,2]$ shows that the quantitative value of the main characteristics of the proton beam extracted at 50 and $70 \mathrm{GeV}$ energy, i.e. maxima of intensity, it's "initial" levels $\left(N_{i n}\right)_{E}$ and gradients of increase $\left(\frac{d I}{d R}\right)_{E}$ are approximately proportional to the coefficient $\gamma_{E}=\frac{70}{50}$ that expresses the primary beam energies relation. 


\section{EVALUATION OF USING THE METHOD ON OTHER ACCELERATORS}

\subsection{FNAL}

Let us suppose, that the relations shown above will be valid also under analogous experiments at higher energies. Then, e.g. for the Tevatron, we would have $\left(\gamma_{E}=\right.$ $800 / 70 \approx 11.4$ ):

- initial level of intensity

$\left(N_{\text {in }}\right)_{800}=\gamma_{E} \cdot\left(N_{\text {in }}\right)_{70} \approx 4.7 \cdot 10^{7}$ particles,

- gradient of intensity increase

$\left(\frac{d I}{d R}\right)_{800}=11.4 \cdot\left(\frac{d I}{d R}\right)_{70} \approx 1.1 \cdot 10^{7}$ particles $/ \mathrm{mm}$.

Therefore, the maximum number of particles extracted by bent crystal may be

$\left(N_{\text {max }}\right)_{800}=\left(N_{\text {in }}\right)_{800}+\left(\frac{d I}{d R}\right)_{800} \cdot \Delta R=9.8 \cdot 10^{7}$ particles, where $\Delta R=5 \mathrm{~mm}$ (see fig.1).

This value of intensity nearly corresponds to the required one for the SFT experiment in SSC and is enough for a scaling experiment on proton extraction by bent crystal which is being prepared in Tevatron [3] and SPS [4].

\section{$3.2 J I N R$}

Let us give analogous estimates for the well known experiment at Dubna [5], where protons with energies 4.2,6.0 and $7.5 \mathrm{GeV}$ were extracted for the first time. Results of estimates for the energies 4.2 and $6.0 \mathrm{GeV}$ are given in Table. Data for the $7.5 \mathrm{GeV}$ energy are not analyzed because the beam line, calculated for the $6 \mathrm{GeV}$ beam, was not able to transport to the experimental hall $7.5 \mathrm{GeV}$ particles.

Table 1: Estimates of possible intensity under using the thin target.

\begin{tabular}{|l|c|c|c|c|}
\hline & $\begin{array}{c}\mathrm{E}, \\
\mathrm{GeV}\end{array}$ & $\begin{array}{c}\text { Intensity under } \\
\text { direct extraction } \\
\text { by crystal [5] }\end{array}$ & $\gamma_{E}$ & $\begin{array}{c}\text { Possible intensity } \\
N_{\max }= \\
\gamma_{E} \cdot\left(N_{\max }\right)_{\text {IHEP }}\end{array}$ \\
\hline JINR & $\mathbf{4 . 2}$ & $(2-5) \cdot 10^{5}$ & 0.06 & $\sim 6 \cdot 10^{5}$ \\
\cline { 2 - 5 } & 6.0 & $\sim 5 \cdot 10^{5}$ & 0.085 & $\sim 8.5 \cdot 10^{5}$ \\
\hline IHEP $^{1}$ & 70.0 & $\sim 4.5 \cdot 10^{6}$ & 1 & $10^{7}$ \\
\hline
\end{tabular}

It is seen that, in case of a thin target use, extracted intensity could be higher, but an influence of scattering effect is expressed weakly. Apparently, this is explained by both large dimensions of a beam at low energy and weak focusing and a small angular divergence of particles of large amplitudes. Additional scattering renders only weak influence on amplitudes and angular divergence of such a beam. At high energies and strong focusing, effects of particle scattering before steering them onto the crystal are expressed more distinctly.

The results of the above made estimates for graphic reason are presented in Figs.2 and 3. Curve 2 of Fig.2 shows the experimental dependence of intensity of the $70-\mathrm{GeV}$ proton beam extracted into a beam line by the bent crystal under the particles scattering with a thin target, while

\footnotetext{
${ }^{1}$ The experimental data.
}

curves 1 and 3 present the prognosis of possible dependencies for the JINR (Dubna) and FNAL accelerators. In Fig.2 $N_{\max }(C R)_{6,70}$ are the levels of maximum intensity extracted from the accelerators JINR and IHEP under the direct steering a beam onto the crystal with the energy 6 and $70 \mathrm{GeV}$ (experiment), respectively.

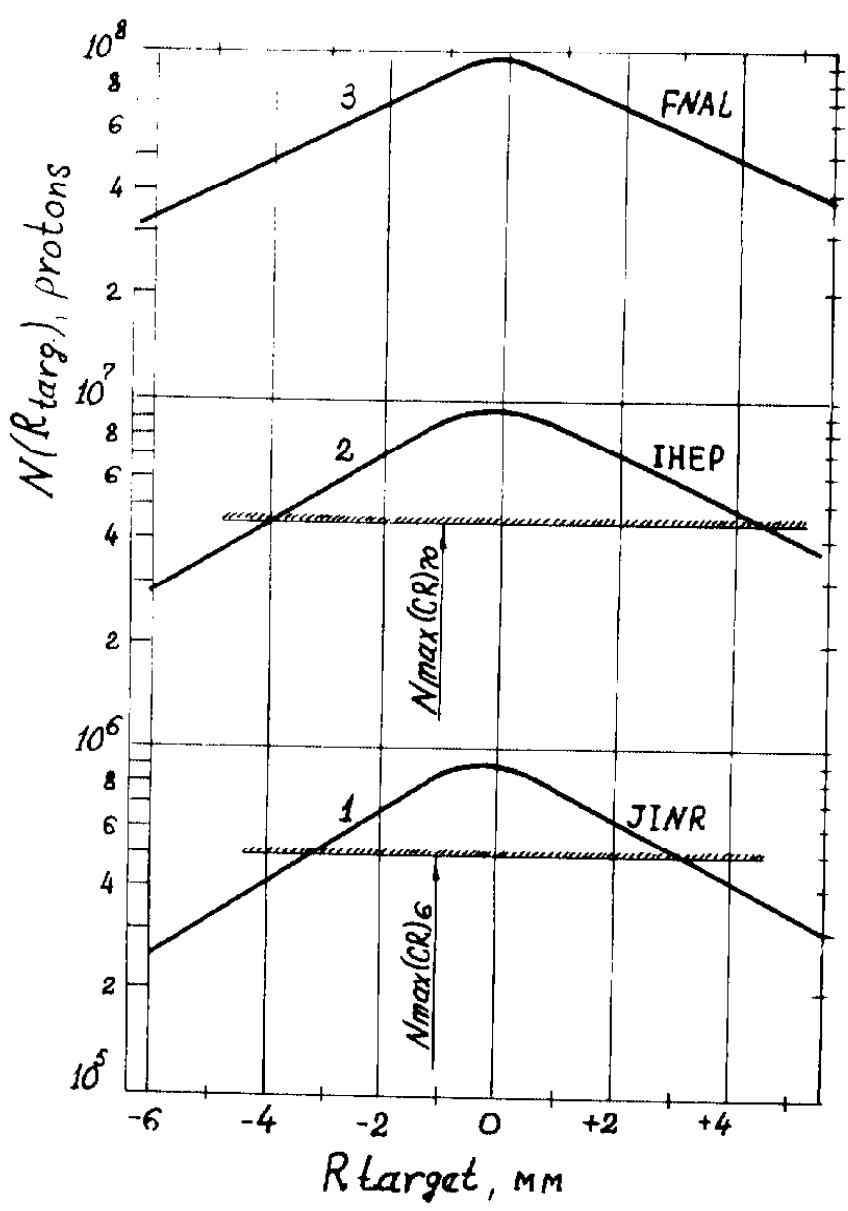

Figure 2: Intensity of the proton beam extracted by bent crystal with use of thin target versus its coordinate. 2 .. IHEP experiment [1], 1,3 - the estimates for the JINR accelerator (Dubna) and Tevatron (FNAL), respectively.

Fig.3 illustrates dependence of maximum intensity extracted by a bent crystal versus accelerated beam energy both for the case of direct steering a beam onto the crystal (curve 1) and after scattering it with the thin target (curve 2). The experimental values are marked with a cross. 


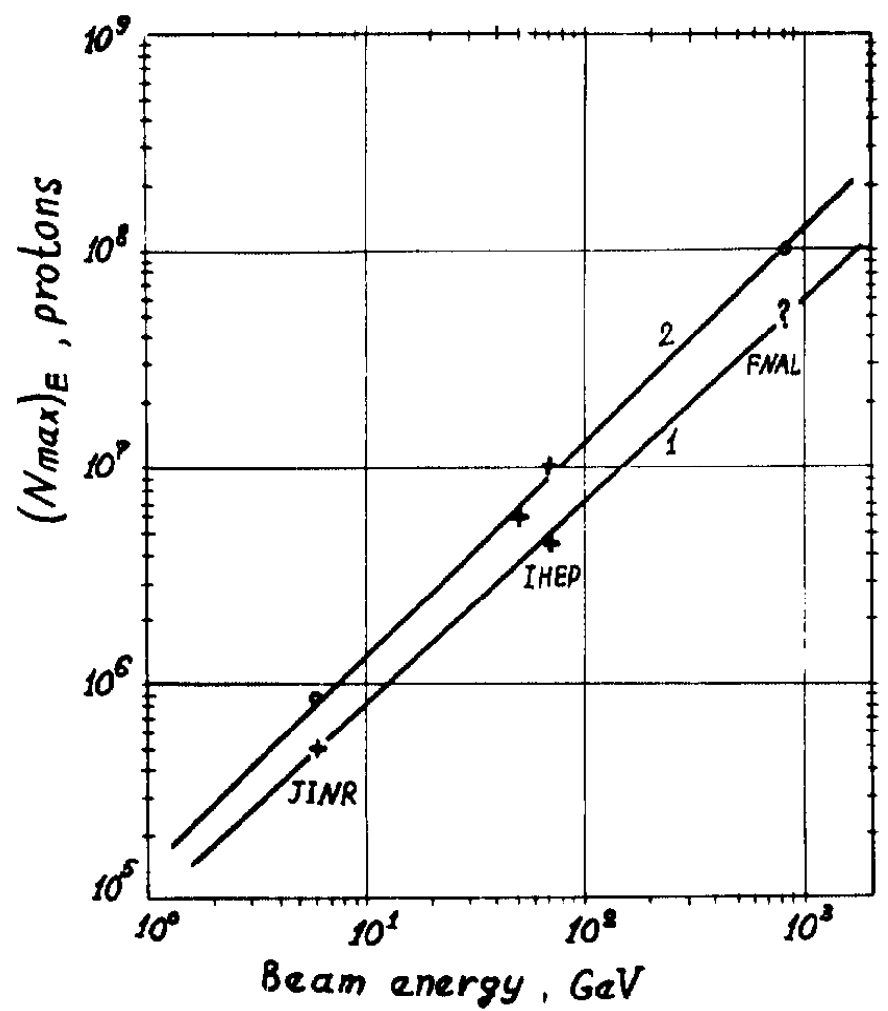

Figure 3: Maximum intensity of the beam extracted by bent crystal versus an energy. 1 - under direct steering a beam onto the crystal, 2 - after scattering with a thin target. The experimental values are marked with a cross.

\section{COINCLUSION}

The data given in $[1,2]$ confirm that the preliminary scattering of particles with a thin target stimulates an increase of the bent crystal extraction efficiency. Extraction efficiency could be obtained with this method may significantly surpass the efficiency attainable under direct steering the beam onto the crystal. Estimates of the extracted beam intensity, that one can suppose in the scaling experiments on the higher energy accelerators, may be checked experimentally.

\section{REFERENCES}

[1] A.A.Asseev et al., IHEP 91-182, Protvino, 1991. To be published in Nucl. Instr. and Meth., Section A.

[2] A.A.Assecv et al., IHEP 92-137, Protvino, 1992.

[3] R.A.Carrigan, Jr. et al. FNAL proposal P-853 (1991).

[4] B.N.Jensen et al. CERN proposal CERN/DRDC 91-25 (1991).

[5] V.V.Avdeichikov et al., JINR Communication 1-84, Dubna, 1984. English translation Fermilab FN-429 (1986). 